\title{
Outer Automorphism Group of the Ergodic Equivalence Relation Generated by Translations of Dense Subgroup of Compact Group on its Homogeneous Space
}

By

\author{
Sergey L. GEFTER *
}

\begin{abstract}
We study the outer automorphism group Out $R_{\Gamma}$ of the ergodic equivalence relation $R_{\Gamma}$ generated by the action of a lattice $\Gamma$ in a semisimple Lie group on the homogeneos space of a compact group $K$. It is shown that Out $R_{\Gamma}$ is locally compact. If $K$ is a connected simple Lie group, we prove the compactness of Out $R_{\Gamma}$ using the D. Witte's rigidity theorem. Moreover, an example of an equivalence relation without outer automorphisms is constructed.
\end{abstract}

\section{Introduction}

An important problem in the theory of full factors [Sak], [Con 1] and in the orbit theory for groups with the $T$-property $[\mathrm{Kaz}]$ is that of studying the group of outer automorphisms of the corresponding object as a topological group. Unlike the amenable case, the outer automorphism group is a Polish space in the natural topology (see Section 1) and its topological properties are algebraic invariants of the factor and orbital invariants of the dynamical system. The first results in this sphere were obtained by A. Connes [Con 2] in 1980 who showed that the outer automorphism group of the $\mathrm{II}_{1}$-factor of an ICC-group with the $T$-property is discrete and at most countable. Various examples of factors and ergodic equivalence relations with locally compact groups of outer automorphisms were constructed in [Cho 1], [EW], [GGN], [Gol], [GG 1] and [GG 2]. In [Gol] and [GG 2] the topological properties of the outer automorphism group were used to construct orbitally nonequivalent

Communicated by H. Araki, November 10, 1995.

1991 Mathematics Subject Classification: 28D15.

The work was supported in part by the International Science Foundation Grant U2B000.

*Kharkov State University, Department of Mechanics and Mathematics, Freedom sq. 4, Kharkou, 310077, Ukraine. 
ergodic actions of arithmetic groups with the $T$-property. The group of outer automorphisms was first calculated in the explicit form in $[G G 1]$ for the equivalence relation given by translations of $S O(n, \mathbb{Q})$ on $S O(n, \mathbb{R}), n \geq 5$ (see Section 4 [GG 2]).

The present paper deals with studying the group of outer automorphisms of the ergodic equivalence relation generated by the translations of a lattice in a semisimple Lie group on the homogeneous space of compact group. Section 1 presents a preliminary information on the groups of outer automorphisms of equivalence relations. Section 2 contains the proof of the general theorem about the local compactness of outer automorphism group of the equivalence relation generated by the action of a group with the $T$-property on the homogeneous space of an arbitrary compact group (Theorem 2.3). As it is shown in the example of the action of $S L(n, \mathbb{Z})$ on $S L\left(n, \mathbb{Z}_{p}\right)$, the group of outer automorphisms can be noncompact (Remark 2.8).

The most important case where the lattice acts on a homogeneous space of a connected conpact simple Lie group is considered in Section 3. In this case the D. Witte's results [Wit] play an important role. We use them to obtain the explicit description of all automorphisms of the equivalence relation (Theorem 3.3). It is proved that the outer automorphisms group is compact, Moreover, we find some conditions which ensure this group to be finite (Corollary 3.4) and trivial (Corollary 3.5). Section 4 involves one of the principal results of the present paper, namely the construction of equivalence relations of type $\mathrm{II}_{1}$ and $\mathrm{II}_{\infty}$ without outer automorphisms (Theorem 4.2 and Corollary 4.3). It shoud be noted that the problem of the existence of a type II factor and type III equivalence relation without outer automorphisms remains unsolved.

The main results are reported in [Gef].

The author is grateful to V. Ya. Golodets and all the participants of Kharkov Seminar on the ergodic theory and operator algebras for their kind attention and helpful discusions of the problems considered in the paper.

\section{\$1. Preliminaries}

Let $\Gamma$ be a countable group, $(X, \nu)$ a free ergodic $\Gamma$-space with finite invariant measure. Denote the equivalence relation generated by $\Gamma$ as $R_{\Gamma}$. Let Aut $R_{\Gamma}$ be the group of its automophisms, Int $R_{\Gamma}$ the inner automorphism subgroup [FM 1], $M=M\left(R_{\Gamma}\right)$ the von Neumann algebra constructed by $R_{\Gamma}$ [FM 2], and $A=L^{\infty}(X, \nu)$. We endow Aut $M$ with the topology of pointwise convergence in norm on $M_{*}$ [Haa]. Then

$$
\operatorname{Aut}(M, A)=\{\theta \in \operatorname{Aut} M: \theta(A)=A\}
$$


and

$$
Z(M, A)=\{\theta \in \operatorname{Aut} M: \theta(a)=a, a \in A\}
$$

are closed (hence Polish) subgroups of Aut $M$. Since Aut $R_{\Gamma}$ can be identified with $\operatorname{Aut}(M, A) / Z(M, A)[\mathrm{FM} 2]$, it is a Polish group itself.

Remark 1.1. Consider the natural $\sigma$-finite measure $m$ using the set $R_{\Gamma}$ [FM 1] and associate the unitary operator $U_{\theta}$ in the space $L^{2}\left(R_{\Gamma}, m\right)$ with the automorphism $\theta$ of $\operatorname{Aut}_{\Gamma}$, by

$$
\left(U_{\theta} \xi\right)(x, y)=\xi\left(\theta^{-1}(x), \theta^{-1}(y)\right)
$$

It can be shown that this topology on $\mathrm{Aut} R_{\Gamma}$ is induced by the weak operator topology when Aut $R_{\Gamma}$ is embedded into the unitary group of $L^{2}\left(R_{\Gamma}, m\right)$. Moreover, this topology coincides with the Polish topology on $\operatorname{Aut} R_{\Gamma}$ which was considered by T. Hamachi and M. Osikawa [HO] (see [Dan, section 3]).

If the group $\Gamma$ is amenable then $R_{\Gamma}$ is an approximately finite equivalence relation $[\mathrm{CFW}]$ and the group $\operatorname{Int} R_{\Gamma}$ of its inner automorphisms is dense in Aut $R_{\Gamma}$ [HO]. The $T$-property is diametrically opposite to the amenability [Kaz, Zim 4]. If $\Gamma$ contains an ergodic subgroup $\Gamma_{0}$ with the $T$-property c.nd $\Gamma$ is an ICC-group with respect to $\Gamma_{0}$, i.e. the set

$$
\left\{\gamma g \gamma^{-1}: \gamma \in \Gamma_{0}\right\}
$$

is infinite for all $g \neq e$, then $\operatorname{Int} R_{\Gamma}$ is a closed subgroup of Aut $R_{\Gamma}[\mathrm{GG} 1, \mathrm{GG} 2]$. Thus, the group of outer automorphisms

$$
\text { Out } R_{\Gamma}=\mathrm{Aut} R_{\Gamma} / \operatorname{Int} R_{\Gamma}
$$

of $R_{\Gamma}$ is a Polish group. It shoud be noted that this result also follows from the more general statement about the closeness of Int $R_{\Gamma}$ in Aut $R_{\Gamma}$ (see [JS, p.113]), since it can be shown that $\Gamma$ with the above properties is not inner ame able [Cho 2, Pr. 6(b)].

For an equivalence relation with the closed subgroup of inner auto-morphisms, the problem of studying the outer automorphism group as a topological group seems natural and important. In the general case the Polish group can be extremely "large".

Example 1.2. Let $(S, \mu)$ be a Lebesgue apace with the probability measure and $\Gamma$ an ICC-group with the $T$-property. Consider the Bernoulli action of $\Gamma$ on the space 


$$
(X, \nu)=\Pi_{r \in \Gamma}(S, \mu)
$$

Since any $\mu$-preserving automorphism of the space $S$ generates an outer automorphism of the equivalence relation $R_{\Gamma}$ the group Out $R_{\Gamma}$ is not locally compact.

In the following section we prove that if $\Gamma$ acts by translations on a homogeneous space of a compact group, then the outer automorphism group of $R_{\Gamma}$ is locally compact.

\section{\$2. The Action of a Countable Dense Subgroup of a Compact Group on its Homogeneous Space}

Let $K$ be a compact group with the Haar measure $\mu, \Gamma$ its countable dense subgroup, and $L$ a closed subgroup of $K$. Consider the action of $\Gamma$ via left translations on the homogeneous space $X=K / L$ with the invariant meaure $\nu$ :

$$
\gamma(k L)=\gamma k L, \quad k \in K, \quad \gamma \in \Gamma
$$

Since $\Gamma$ is dense in $K$, we have that $(X, \nu)$ is an ergodic $\Gamma$-space.

Lemma 2.1. Assume that $K$ is a compact connected simple Lie group with the trivial center. Then the action of $\Gamma$ on $(X, \nu)$ is free.

Proof. Consider the following sets for $\gamma \in \Gamma$

$$
\begin{aligned}
& S \gamma=\{x \in X: \gamma x=x\} \text { and } \\
& T \gamma=\{k \in K: \gamma k L=k L\} .
\end{aligned}
$$

Assume that $\nu(S \gamma)>0$. Then $\mu(T \gamma)>0$. Since $K$ and $L$ are real algebraic groups, the set $T_{r}$ is a real algebraic subvariety in $K$. Consequently, $T_{r}=K$. Hence

$$
\gamma \in \widetilde{L}=\bigcap_{k \in K} k L k^{-1}
$$

One can easily see that $\widetilde{L}$ is a normal subgroup in $K$. Consequently, $\widetilde{L} \subset Z(K)$ $=\{e\}$, i.e. $\gamma=e$.

Let $N_{K}(L)$ be the normalizer of $L$ in $K$. If $t \in N_{k}(L)$, then the right translation $\gamma_{t}$ is well defined on $(X, \nu)$, as follows: 


$$
r_{t}(x)=x t^{-1}, \quad x \in X
$$

It is obvious that $r_{t}=\mathrm{id}$ if and only if $t \in L$. Moreover, the automorphism $r_{t}$ lies in the centralizer of the $\Gamma$-action.

Lemma 2.2. The centralizer of the $\Gamma$-action coincides with the group of right translations, i.e. isomorphic to $N_{K}(L) / L$.

Proof. Assume the Borel map $\beta: X \rightarrow X$ commutes with left translations of $\Gamma$. Denote by $p$ the projection from $K$ onto $X=K / L$ and consider the map

$$
\phi: K \rightarrow X, \phi(k)=k^{-1} \beta(p(k))
$$

According to the conditions, $\beta(\gamma x)=\gamma \beta(x)$ for all $\gamma \in \Gamma$ at a.a. $x \in X$. Consequently, $\phi(\gamma k)=\phi(k)$ for all $\gamma \in \Gamma$ and a.a. $k \in K$. Since the $\Gamma$-action is ergodic on $(K, \mu)$ there are $t \in K$ and $K_{0} \subset K$ so that $\mu\left(K_{0}\right)=1$ and $\phi(k)=$ $p\left(t^{-1}\right)$ for all $k \in K_{0}$. Therefore

$$
\beta(p(k))=k \phi(k)=p\left(k t^{-1}\right), \quad k \in K_{0}
$$

We show that $t \in N_{k}(L)$. Let $h \in L$. Since $\mu\left(K_{0} h^{-1} \cap K_{0}\right)=1$, there is an element $k \in K_{0}$ with $k h \in K_{0}$. Then

$$
\begin{aligned}
\beta(p(k)) & =\beta(p(k h))=k h p\left(t^{-1}\right), \text { i.e. } \\
p\left(t^{-1}\right) & =h p\left(t^{-1}\right) \text { and } t h t^{-1} \in L .
\end{aligned}
$$

Thus, $t L t^{-1} . \subset L$. Using the compactness of $K$ one can demonstrate that $t^{-1} L t \subset$ $L$, i.e. $t \in N_{K}(L)$. Now, in view of (2.1), $\beta(x)=r_{t}(x)$ for a.a. $x \in X$.

Consider the ergodic equivalence relation $R_{\Gamma}$ generated by left translations of $\Gamma$ on $(X, \nu)$ and denote by $\varepsilon$ the projection from $\operatorname{Aut} R_{\Gamma}$ outo $\operatorname{Out} R_{\Gamma}$. The following theorem about the topological structure of Out $R_{\Gamma}$ is a generalization of the results of Section 3 in [GG 2], where the case $L=\{e\}$ is considered.

Theorem 2.3. Assume that $\Gamma$ acts freely on $(X, \nu)$ and contains a dense of subgroup $\Gamma_{0}$ in $K$ with the T-property, and $\Gamma$ is an ICC-group with respect to $\Gamma_{0}$, i.e. the set $\left\{\gamma g \gamma^{-1}: \gamma \in \Gamma_{0}\right\}$ is infinite for all $g \neq e$. Then $\varepsilon\left(\left\{\gamma_{t}: t \in N_{K}(L)\right\}\right)$ is an open subgroup in Out $R_{\Gamma}$, which is topologically isomorphic to $N_{k}(L) / L$. Hence, Out $R_{\Gamma}$ is a locally compact group.

To prove Theorem 2.3, we shall provide several considerations. 
Given $\gamma \in \Gamma$ and $t \in N_{k}(L)$, we denote by $\tilde{l}_{\gamma}$ and $\tilde{r}_{t}$ the automorphisms of $A=L^{\infty}(X, \nu)$ generated by $\gamma$-left and $t$-right translations:

$$
\tilde{l}_{\gamma}(a)(x)=a\left(\gamma^{-1} x\right), \tilde{r}_{t}(a)(x)=a(x t), a \in A, x \in X .
$$

Consider the crossed product $M=W^{*}\left(A, \tilde{l}_{\gamma}, \Gamma\right)$. The algebra $M$ is generated by operators $\pi(a)$ and $\lambda_{\gamma}(a \in A, \gamma \in \Gamma)$ of the space $L^{2}(X, \nu) \otimes l^{2}(\Gamma)$ given by

$$
\begin{aligned}
(\pi(a) \xi)(x, g) & =a(g x) \xi(x, g), \\
\left(\lambda_{r} \xi\right)(x, g) & =\xi\left(x, \gamma^{-1} g\right) .
\end{aligned}
$$

Then

$$
\pi\left(\tilde{l}_{r}(a)\right)=\lambda_{r} \pi(a) \lambda_{r}^{*}, a \in A, r \in \Gamma
$$

Since $(X, \nu)$ is a free $\Gamma$-space, the algebra $M=W^{*}(A, l, \Gamma)$ is isomorphic to the algebra $M\left(R_{\Gamma}\right)$ constructed by $R_{\Gamma}$. Set up

$$
C_{\Gamma 0}=\left\{\theta \in \operatorname{Aut} M: \theta\left(\lambda_{r}\right)=\lambda_{r}, \gamma \in \Gamma\right\}
$$

Lemma 2.4. If $\theta \in C_{\Gamma_{0}}$, then the automorphism $\theta$ maps the algebra $A$ into itself, i.e.

$$
C_{\Gamma_{0}} \subset \operatorname{Aut}(M, A)
$$

Proof. Let $\widehat{K}$ be the dual space of the group $K$. Denote by $\widehat{K}_{L}$ the set of irreducible representations $\sigma \in \widehat{K}$ which admit a nontrivial $L$-invariant vector in the space of representations $H_{\sigma}$. For each $\sigma \in \widehat{K}$ we select an orthonormal basis $\left\{\xi_{i}^{\sigma}: i=1, \cdots, \operatorname{dim} \sigma\right\}$ of $H_{\sigma}$ in such a way that the first $q_{\sigma}$ its elements form a basis of the space of $L$-invariant vectors. Determine the matrix elements of the representation $\sigma$ as

$$
\sigma_{i j}(k)=\left(\sigma(k) \xi_{j}^{\sigma} \mid \xi_{j}^{\sigma}\right), \quad i, j=1, \cdots, \operatorname{dim} \sigma .
$$

Then $\sigma_{i j}(k h)=\sigma_{i j}(k)$ if $h \in L, k \in K$ and $j \leq q_{\sigma}$. Consequently, for $j \leq q_{\sigma}$ the matrix elements $\sigma_{i j}$ can be considered as functions on the homogeneous space $X$ $=K / L$. The system

$$
\left\{\sigma_{i j}: \sigma \in \widehat{K}_{L}, \quad i=1, \cdots, \operatorname{dim} \sigma, \quad j=1, \cdots, q_{\sigma}\right\}
$$


is an orthogonal basis in $L^{2}(X, \nu)$ with $\sigma_{\imath j} \in A=L^{\infty}(X, \nu)$.

For $\gamma \in \Gamma$ we have

$$
\sigma_{i j}\left(\gamma^{-1} k\right)=\sum_{p=1}^{\operatorname{dim} \sigma} \sigma_{i p}\left(\gamma^{-1}\right) \sigma_{p j}(k), \text { i.e. } \tilde{l}_{r}\left(\sigma_{i j}\right)=\sum_{p=1}^{\operatorname{dim} \sigma} \overline{\sigma_{p i}(\gamma)} \sigma_{p j}
$$

Let $\theta \in C_{\Gamma_{0}}$ and

$$
\theta\left(\pi\left(\sigma_{i j}\right)\right)=\sum_{g \in \Gamma} \sum_{\rho \in \widehat{K}_{L}} \sum_{m=1}^{\operatorname{dim} \rho} \sum_{n=1}^{q \sigma} c(g ; m, n, \rho) \pi\left(\rho_{m n}\right) \lambda_{g} .
$$

Since the algebra $A$ is generated by the matrix elements $\sigma_{i j}$, it suffices to prove that

$$
\begin{aligned}
& \theta\left(\pi\left(\sigma_{i j}\right)\right) \in \pi(A), \sigma \in \widehat{K}_{L}, \\
& i=1, \cdots, \operatorname{dim} \sigma, \mathrm{j}=1, \cdots, q_{\sigma} .
\end{aligned}
$$

By (2.3), the equality

$$
\lambda_{r} \theta\left(\pi\left(\sigma_{i j}\right)\right) \lambda_{r}^{*}=\theta\left(\pi\left(\hat{l}_{r}\left(\sigma_{i j}\right)\right)\right), \gamma \in \Gamma_{0}
$$

the ICC-property, and convergence of series (2.4), we can obtain $c(g ; m, n, \rho)$ $=0$ for $g \neq e$ and hence, $\theta\left(\pi\left(\sigma_{i j}\right)\right) \in \pi(A)$.

For $t \in N_{K}(L)$ denote by $R_{t}$ the automorphism of the factor $M$ given by

$$
R_{t}(\pi(a))=\pi\left(\widetilde{r}_{t}(a)\right), \quad R_{t}\left(\lambda_{\gamma}\right)=\lambda_{\gamma}, a \in A, \quad \lambda \gamma \in \Gamma
$$

Besides, given a cocycle $c \in Z^{1}(\Gamma, U(A))$ we define an automorphism $\theta_{c}$ of $M$ by setting

$$
\theta_{c}(\pi(a))=\pi(a), \theta_{c}\left(\lambda_{r}\right)=\pi\left(c_{r}\right) \lambda_{r}
$$

(see [FM 2]).

Lemma 2.5. Let $\theta \in C_{\Gamma 0}$. Then there exist $t \in N_{K}(L)$ and $c \in Z^{1}(\Gamma, U(A))$ such that $\theta=\theta_{c} R_{t}$. The cocycle $c$ here is defined uniquely while $t$ is defined up to an element of $L$.

Proof. In view of Lemma 2.4, $\theta$ determines the automorphism $\widetilde{\beta}$ of the algebra $A: \pi \circ \widetilde{\beta}=\theta \circ \pi$. By the assumption, $\theta\left(\lambda_{r}\right)=\lambda_{r}$ and so $\widetilde{\beta} \widetilde{\lambda}_{r}=\widetilde{\lambda}_{r} \widetilde{\beta}, \gamma$ $\in \Gamma$. According to 2.2, $\widetilde{\beta}=\widetilde{r}_{t}$ for a some $t \in N_{K}(L)$. Set up $\theta_{1}=\theta R_{t}^{-1}$. 
Then $\theta_{1}(\pi(a))=\pi(a), a \in A$. Therefore there is $c \in Z^{1}(\Gamma, U(A))$, such that $\theta_{1}=\theta_{c}\left(\right.$ see $[\mathrm{FM} \mathrm{2]})$. Hence, $\theta=\theta_{c} R_{t}$.

Lemma 2.6. Let $\Gamma$ be an ICC-group, $(X, \mu)$ a free ergodic $\Gamma$-space with $f i$ nite invariant measure and $\theta: X \rightarrow X$ an automorphism such that $\theta(\gamma x)=\gamma \theta(x)$. If $\theta \neq \mathrm{id}$, then $\theta$ is an outer automorphism of the equivalence relation $R_{\Gamma}$.

Proof. Suppose that $\theta \in \operatorname{Int} R_{\Gamma}$. Set up

$$
E_{\alpha}=\{x \in X: \theta(x)=\alpha x\}
$$

Then $\gamma E_{\alpha}=E_{\gamma \alpha \gamma^{-1}}$ and, hence,

$$
\mu\left(E_{\gamma \alpha r^{-1}}\right)=\mu\left(E_{\alpha}\right), \gamma \in \Gamma \text {. }
$$

Since by our assumption $\theta \in \operatorname{Int} R_{\Gamma}$, there is $\gamma_{0} \in \Gamma$ with $\mu\left(E_{r_{0}}\right)>0$. However, the set $\left\{\gamma \gamma_{0} \gamma^{-1}: \gamma \in \Gamma\right\}$ is infinite if $\gamma_{0} \neq e$. Therefore $\gamma_{0}=e$. Consequently, $\gamma E_{e}=E_{e}$ for all $\gamma \in \Gamma$, i.e., $\mu\left(E_{e}\right)=\mu(X)$ and $\theta=$ id.

\section{Proof of Theorem 2.3.}

Denote by $C_{T 0}^{M}$ the subgroup of Aut $M$ which is algebraically generated by $C_{\Gamma_{0}}$ and Int $M$. According to 2.3 [GG 2], $C_{\Gamma_{0}}^{M}$ is an open subgroup of Aut $M$. Therefore $C_{\Gamma_{0}}^{M} \cap \operatorname{Aut}(M, A)$ is an open subgroup of $\operatorname{Aut}(M, A)$. Furthermore, in view of Lemma $2.4, C_{\gamma_{0}} \subset \operatorname{Aut}(M, A)$ and, hence, the subgroup $C_{\Gamma_{0}}^{M} \cap$ Aut $(M, A)$ is generated by $C_{\Gamma_{0}}$ and $\operatorname{Int}(M, A)=\operatorname{Aut}(M, A) \cap \operatorname{Int} M$. Using Lemma 2.5 we deduce that the subgroup generated algebraically by $\operatorname{Int}(M, A), Z(M, A)$ $\approx Z^{1}(\Gamma, U(A))$, and $\left\{R_{t}: t \in N_{K}(L)\right\}$ is open in $\operatorname{Aut}(M, A)$. Consequently, in view of Lemma 2.1 [GG 2], the subgroup generated by $\operatorname{Int} R_{\Gamma}$ and $\left\{r_{t}: t \in N_{k}(L)\right\}$ is open in $\mathrm{Aut} R_{\Gamma}$. Now it should be noted that

$$
\left\{r_{t}: t \in N_{K}(L)\right\} \cap \operatorname{Int} R_{\Gamma}=\{\mathrm{id}\}
$$

(see Lemma 2.6). Thus, $\varepsilon\left(\left\{r_{t}: t \in N_{k}(L)\right\}\right)$ is an open subgroup of Out $R_{\Gamma}$ isomorphic algebraically to $N_{K}(L) / L$. Since Out $R_{\Gamma}$ is a Polish group and $\varepsilon$ is continuous, the groups $\varepsilon\left(\left\{r_{t}: t \in N_{k}(L)\right\}\right)$ and $N_{k}(L) / L$ are topologicaly isomorphic.

Remark 2.7. Note the two significant cases where the group $\Gamma$ is an ICC-group with respect to the subgroup $\Gamma_{0}$ [GG 2, 1.6 and 3.7]:

(a) $\Gamma$ is isomorphic to the subgroup in the connected semisimple Lie group with trivial center and without compact factors, $\Gamma_{0}$ being a lattice in this 
Lie group;

(b) $K$ is a connected group with trivial center.

Remark 2.8. The group Out $R_{\Gamma}$ can be noncompact. For example, let

$$
K=S L\left(n, \mathbb{Z}_{p}\right), \Gamma=S L(n, \boldsymbol{Z}), n=2 j+1 \geq 3, L=\{e\}
$$

( $\mathbf{Z}_{p}$ is the ring of $p$-adic integers). It can be shown that Out $R_{\Gamma}$ contains an open subgroup which is topologically isomorphic to the group $H=S L\left(n, \mathbf{Q}_{p}\right)$. Let $\Lambda$ $=S L\left(n, \mathbb{Z}\left[\frac{1}{p}\right]\right)$. Then $\Lambda$ is a dense subgroup in $H$ and $K \cap \Lambda=\Gamma$. Consider the ergodic equivalence relation $R_{\Lambda}$ of the type $\Pi_{\infty}$ generated by left translations of $\Lambda$ on the space $(H, \tilde{\mu})$ where $\tilde{\mu}$ is the Haar mesure on $H$. Since $K$ is an open subgroup in $H, R_{\Lambda} \approx R_{\Gamma} \times I_{\infty}$ where $I_{\infty}$ is the transitive equivalence relation on $H / K$. Besides, the map $\phi: K \times H / K \rightarrow H$ which is responsible for isomorphism of $R_{\Gamma} \times I_{\infty}$ and $R_{\Lambda}$ can be chosen so that

$$
\phi\left(r_{t} \times \mathrm{id}\right) \phi^{-1}=r_{t} \text { for } t \in K
$$

For $h \in H$, denote the right translation on the space $(H, \widetilde{\mu})$ by $r_{h}: v_{h}(s)=s h^{-1}$, $s \in H$. We claim that if $h \neq e, r_{h}$ is an outer automorphism of $R_{\Lambda}$. In fact, assume that $r_{h} \in \operatorname{Int} R_{\Lambda}$. Then there exists $\alpha \in \Lambda$ for which the set $E_{\alpha}=\{s \in$ $\left.H: s h^{-1}=\alpha s\right\}$ has the positive measure. Consider the closed subgroup $F_{\alpha}$ generated by the set $\left\{s_{2}^{-1} s_{1}: s_{1}, s_{2} \in E_{\alpha}\right\}$. If $s_{1}, s_{2} \in E_{\alpha}$, then $s_{1} h^{-1}=\alpha s_{1}, h s_{2}^{-1}=$ $s_{2}^{-1} \alpha^{-1}$, and hence $h s_{2}^{-1} s_{1} h^{-1}=s_{2}^{-1} s_{1}$, i. e. $h$ lies in the subgroup $F_{\alpha}$ centralizer. Since $F_{\alpha}$ is a closed subgoup of positive measure, $F_{a}$ is open in $H$. However, in this case $F_{\alpha}$ contains an open compact subgroup of the form $K(m)=\overline{\Gamma(m)}$, where

$$
\Gamma(m)=\left\{\gamma \in \Gamma: \equiv 1\left(\bmod p^{m}\right)\right\}
$$

Here $\Gamma(m)$ is the lattice in $S L(n, \mathbf{R})$. Consequently, groups $\Gamma(m)$ and $F_{\alpha} \supset$ $\Gamma(m)$ are Zariski dense in $H=S L\left(n, \mathbf{Q}_{p}\right)$ [Wan]. Hence, we obtain that $h \in$ $Z(H)$, and it means that $h=\alpha^{-1} \in \Lambda$, i.e. $h \in Z(\Lambda)=\{e\}$. Thus, $H$ is embedded into Out $R_{\Lambda} \approx \operatorname{Out}\left(R_{\Gamma} \times \mathrm{I}_{\infty}\right) \approx \operatorname{Out} R_{\Gamma}$ (see [GG 2, Corollary B.3]).

It should be noted that if $K$ is a connected group and $L=\{e\}$, Out $R_{\Gamma}$ is a compact group [GG 2, Theorem 4.9]. Using the D. Witte's results [Wit] the following section will give a proof of the compactness of Out $R_{\Gamma}$ for the action of $\Gamma$ on a homogeneous space of a connected Lie group. 


\section{\$3. Automorphisms of the Equivalence Relation Generated by Lattice Translations in the Lie Group on a Homogeneous Space of a Compact Lie Group}

This Section is concerned with automorphisms of the equivalence relation constructed by the $\Gamma$-action on the space $X=K / L$ in the most important case where $\Gamma$ is a lattice in a simple Lie group and $K$ a compact simple Lie group. We obtain the explicit description of automorphisms (Theorem 3.3) using the D. Witte's argument [Wit, 9.4].

We need the following general statement which can be deduced from $\mathrm{R}$. Zimmer's rigidity theorem [Zim 2, 4.3 and 4.5].

Proposition 3.1. Let $G$ be a connected noncompact simple Lie group with trivial center and $\mathbb{R}$-rank $(G) \geq 2, \Gamma$ a lattice in $G$ and $(X, \nu)$ a free ergodic $\Gamma$-space with the finite invariant measure. Let $\theta \in$ Aut $R_{\Gamma}$ and $\alpha: X \times \Gamma \rightarrow \Gamma$ be the cocycle corresponding to $\theta$, i.e. $\theta(\gamma x)=\alpha(x, \gamma) \theta(x)$ for all $\gamma \in \Gamma$ and a. $a$. $x$ $\in X . \quad$ Then there exist Borel function $\phi: X \rightarrow G$ and $\sigma_{1} \in$ Aut $G$ such that $\alpha(x, \gamma)$ $=\phi(\gamma x)^{-1} \sigma_{1}(\gamma) \phi(x)$.

Proof. Using the action of $\Gamma$, let us construct that of $G$ (the induced action $\left[\right.$ Zim 1]) and an automorphism $\dot{\widetilde{\theta}} \in \mathrm{Aut} R_{G}$ corresponding to $\theta \in \mathrm{Aut} R_{\Gamma}$. Let $\pi: G \rightarrow \Gamma \backslash G$ be the natural projection, $\pi(g)=\Gamma g$, and $\omega: \Gamma \backslash G \rightarrow G$ its Borel section, i.e., $\pi \circ \omega=$ id. Determine Borel maps $s: G \rightarrow G$ and $f: G \rightarrow \Gamma$ as follows

$$
s(g)=\omega(\pi(g)), f(g)=g s(g)^{-1}, g \in G .
$$

Set up $S=\omega(\Gamma \backslash G)=s(G)$. Then $S$ is a Borel set, and each element $g$ of $G$ is uniquely represented in the form

$$
g=f(g) s(g), f(g) \in \Gamma, s(g) \in S .
$$

Now consider the diagonal action of $\Gamma$ on the space $X \times G$, i.e.

$$
\gamma(x, h)=(\gamma x, \gamma h), \quad \gamma \in \Gamma, x \in X, h \in G
$$

The orbit partition of $X \times G$ of this action is measurable, and the orbit space $Y$ $=(X \times G) / \Gamma$ can be identified with the space $X \times(\Gamma \backslash G)$ via the Borel isomorphism

$$
\Phi: X \times(\Gamma \backslash G) \rightarrow(X \times G) / \Gamma
$$

given by 


$$
\Phi((x, \pi(h)))=[x, s(h)]
$$

where $[x, h]$ is the image of $(x, h) \in X \times G$ in the space $Y=(X \times G) / \Gamma$. It should be noted that

$$
[\gamma x, \gamma h]=[x, h]=\left[f(h)^{-1} x, s(h)\right], x \in X, h \in G, \gamma \in \Gamma .
$$

Using the isomorphism $\Phi$, we introduce the measure $\rho=\Phi_{*}(\nu \times \lambda)$ on $Y$, where $\lambda$ is the finite $G$-invariant measure on $\Gamma \backslash G$. Now determine the induced action of group $G$ on $(Y, \rho)[\mathrm{Zim} 1]$ :

$$
g[x, h]=\left[x, h g^{-1}\right], \quad x \in X, h \in G
$$

It can be easily verified that $\Phi$ transforms this action to the following action on the space $X \times(\Gamma \backslash G)([\operatorname{Zim} 1,2.2])$ :

$$
g(x, \pi(h))=\left(c(g, \pi(h)) x, \pi(h) g^{-1}\right)
$$

where $c(g, \pi(h))=s\left(h g^{-1}\right) g s(h)^{-1} \in \Gamma, h \in G, x \in X$. Thus, the action of $G$ on $(Y, \rho)$ is free, properly ergodic, and preserves the finite measure $\rho$ [Zim 1,2.7]. Denote by $R_{G}$ the equivalence relation generated by the action of $G$ on $(Y, \rho)$. Let $I$ be the transitive equivalence relation generated by translations of $G$ on $\Gamma \backslash G$. It can be readily seen that $\Phi$ (see (3.2)) carries out isomorphism of $R_{\Gamma} \times I$ and $R_{G}$. Using the automorphism $\theta \in$ Aut $R_{\Gamma}$, one can construct an automorphism $\tilde{\theta} \in R_{G}: \widetilde{\theta}=\Phi(\theta \times \mathrm{id}) \Phi^{-1}$. The direct calculation shows that

$$
\tilde{\theta}([x, h])=\left[\theta\left(f(h)^{-1} x\right), s(h)\right]
$$

(see (3.1)-(3.3)) .

Consider the cocycle $\widetilde{\alpha}: Y \times G \rightarrow G$ associated to $\widetilde{\theta}:$

$$
\widetilde{\theta}(g y)=\widetilde{\alpha}(y, g) \widetilde{\theta}(y), g \in G, y \in Y .
$$

In view of the Zimmer's rigidity theorem, $\widetilde{\alpha}$ is cohomologous to the restriction of an automorphism of $G$ [Zim 2]. Thus, there exist a Borel function $\phi:(X \times G) / \Gamma$ $\rightarrow G$ and $\widetilde{\sigma}_{1} \in$ Aut $G$ are such that

$$
\widetilde{\alpha}(y, g)=\phi^{-1}(g y) \widetilde{\sigma}_{1}(g(\phi(y))
$$

for any $g \in G$ at a.a. $y \in Y$. Set up 


$$
\widetilde{\theta}_{1}(y)=\phi(y) \tilde{\theta}(y), y \in Y .
$$

According to [Zim 2, Prop. 2.4] and [Zim 3, Lemma 3.5], $\widetilde{\theta}_{1}$ is an automorphism of $(Y, \rho)$ and

$$
\tilde{\theta}_{1}(g y)=\widetilde{\sigma}_{1}(g) \tilde{\theta}_{1}(y)
$$

for each $g \in G$ at a.a. $y \in Y$. Herewith, $\widetilde{\theta}_{1} \in \operatorname{Aut} R_{G}$ and $\widetilde{\theta}_{\tilde{\theta}}^{-1} \in \operatorname{Int} R_{G}$. For $g \in G$ and $y=[x, h] \in Y$ we have (see (3.4) and (3.5)):

$$
\begin{aligned}
\tilde{\theta}_{1}(g y) & =\phi(g y) \tilde{\theta}_{1}(g y)=\psi\left(\left[x, h g^{-1}\right]\right) \widetilde{\theta}\left(\left[x, h g^{-1}\right]\right) \\
& =\psi\left(\left[x, h g^{-1}\right]\right)\left[\theta\left(f\left(h g^{-1}\right)^{-1} x\right), s\left(h g^{-1}\right)\right] \\
& =\left[\theta\left(f\left(h g^{-1}\right)^{-1} x\right), s\left(h g^{-1}\right) \psi\left(\left[x, h g^{-1}\right]\right)^{-1}\right]
\end{aligned}
$$

On the other hand,

$$
\begin{aligned}
\widetilde{\sigma}_{1}(g) \widetilde{\theta}_{1}(g) & =\widetilde{\sigma}_{1}(g) \phi([x, h])\left[\theta\left(f(h)^{-1} x\right), s(h)\right] \\
& =\left[\theta\left(f(h)^{-1} x\right), s(h) \phi\left([x, h)^{-1} \widetilde{\sigma}_{1}(g)^{-1}\right]\right.
\end{aligned}
$$

Now we obtain from (3.6)

$$
\begin{aligned}
& {\left[\theta\left(f\left(h g^{-1}\right)^{-1} x, s\left(h g^{-1} \psi\left(\left[x, h g^{-1}\right]\right)^{-1}\right)\right]\right.} \\
& =\left[\theta\left(f(h)^{-1} x\right), s(h) \psi\left([x, h)^{-1} \sigma_{1}(g)^{-1}\right]\right.
\end{aligned}
$$

for all $g \in G$ and a.a. $[x, h] \in Y$.

Since $\theta(\gamma x)=\alpha(x, \gamma) \theta(x)$, we rewrite (3.7) as follows

$$
\begin{aligned}
& {\left[\alpha\left(x, f\left(h g^{-1}\right)^{-1}\right) \theta(x), s\left(h g^{-1}\right) \psi\left(\left[x, h g^{-1}\right]\right)^{-1}\right]} \\
& \quad=\left[\alpha\left(x, f(h)^{-1}\right) \theta(x), s(h) \phi([x, h])^{-1} \widetilde{\sigma}_{1}(g)^{-1}\right]
\end{aligned}
$$

or

$$
\begin{aligned}
& {\left[\theta(x), \alpha\left(x, f\left(h g^{-1}\right)^{-1}\right)^{-1} s\left(h g^{-1}\right) \phi\left(\left[x, h g^{-1}\right]\right)^{-1}\right]} \\
& \quad=\left[\theta(x), \alpha\left(x, f(h)^{-1}\right)^{-1} s(h) \phi([x, h])^{-1} \widetilde{\sigma}_{1}(g)^{-1}\right]
\end{aligned}
$$

Hence, we obtain

or

$$
\begin{aligned}
& \alpha\left(x, f\left(h g^{-1}\right)^{-1}\right)^{-1} s\left(h g^{-1}\right) \psi\left(\left[x, h g^{-1}\right]\right)^{-1} \\
& \quad=\alpha\left(x, f(h)^{-1}\right)^{-1} s(h) \psi([x, h])^{-1} \widetilde{\sigma}_{1}(g)^{-1}
\end{aligned}
$$




$$
\begin{gathered}
\alpha\left(x, f\left(h g^{-1}\right)^{-1}\right)^{-1} s\left(h g^{-1}\right) \psi\left(\left[x, h g^{-1}\right]\right)^{-1} \widetilde{\sigma}_{1}\left(h g^{-1}\right)^{-1} \\
=\alpha\left(x, f(h)^{-1}\right)^{-1} s(h) \psi([x, h])^{-1} \widetilde{\sigma}_{1}(h)^{-1}
\end{gathered}
$$

for all $g \in G$ and a.a. $[x, h] \in Y$. Then for a.a. $x \in X$ (3.8) is satisfied at a.a. $g, h \in G$. Consequently, for a.a. $x \in X$ we have

$$
\alpha\left(x, f(h)^{-1}\right)^{-1} s(h) \psi([x, h])^{-1} \widetilde{\sigma}_{1}(h)^{-1}={ }_{c}(x) \text { at a. a. } h \in G
$$

Since the set $S \subset G$ has positive Haar measure (see (3.1)), it follows that for a. a. $x \in X$

$$
\begin{gathered}
\alpha\left(x, f\left(\gamma^{-1} s(h)\right)^{-1}\right)^{-1} s\left(\gamma^{-1} s(h)\right) \psi\left(\left[x, \gamma^{-1} s(h)\right]\right)^{-1} \widetilde{\sigma}_{1}\left(\gamma^{-1} s(h)\right)^{-1} \\
=\alpha\left(x, f(s(h))^{-1}\right)^{-1} s(s(h)) \psi([x, s(h)])^{-1} \widetilde{\sigma}_{1}(s(h))^{-1}
\end{gathered}
$$

for all $\gamma \in \Gamma$ and a. a. $s(h) \in S$ (we have substituted $h_{1}=\gamma^{-1} s(h)$ and $h_{2}=s$ (h) into (3.9)). Now it should be noted that

$$
f\left(\gamma^{-1} s(h)\right)=\gamma^{-1}, s\left(\gamma^{-1} s(h)\right)=s(h), \phi\left(\left[x, \gamma^{-1} s(h)\right]\right)=\phi([\gamma x, s(h)]) .
$$

Hence,

$$
\begin{gathered}
\alpha(x, \gamma)^{-1} s(h) \psi([\gamma x, s(h)])^{-1} \widetilde{\sigma}_{1}(s(h))^{-1} \widetilde{\sigma}_{1}(\gamma) \\
=s(h) \psi([x, s(h)])^{-1} \widetilde{\sigma}_{1}(s(h)),
\end{gathered}
$$

or

$$
\alpha(x, \gamma)=s(h) \psi([\gamma x, s(h)])^{-1} \widetilde{\sigma}_{1}\left(s(h)^{-1} \gamma s(h)\right) \psi\left([x, s(h)] s(h)^{-1}\right.
$$

for all $\gamma \in \Gamma$ and a. a. $x \in X$ and $h \in G$.

Take an element $s(h) \in S$ such that (3.10) is satisfied for all $\gamma \in \Gamma$ and a. a. $x \in X$ and put

$$
\phi(x)=\phi([x, s(h)]) s(h)^{-1}, \sigma_{1}(g)=\widetilde{\sigma}_{1}\left(s(h)^{-1} g s(h)\right)
$$

Then $\alpha(x, \gamma)=\phi(\gamma x)^{-1} \sigma_{1}(\gamma) \phi(x)$ for all $\gamma \in \Gamma$ and a. a. $x \in X$.

While studying the group Out $R_{\Gamma}$, we need an interesting analogue of Lemma 2.6. 
Lemma 3.2. Let $\Gamma$ be an irreducible lattice in a connected semisimple Lie group $G$ with trivial center, without compact factors, and $\mathbb{R}-\operatorname{rank}(G) \geq 2,(X, \mu)$ a free ergodic $\Gamma$-space with finite invariant measure and $\theta: X \rightarrow X$ an automorphism such that $\theta(\gamma x)=\sigma(\gamma) \theta(x)$ for some $\sigma \in \operatorname{Aut} \Gamma$. If $\theta$ is an inner automorphism of the equivalence relation $R_{\Gamma}$, there is an element $\gamma_{0} \in \Gamma$ such that $\theta(x)=\gamma_{0} x$ and $\sigma(\gamma)=\gamma_{0} \gamma \gamma_{0}^{-1}$.

Proof. We for $\alpha \in \Gamma$ set up $E_{\alpha}=\{x \in X: \theta(x)=\alpha x\}$. Then $\gamma E_{\alpha}=$ $E_{\sigma(\gamma) \alpha \gamma^{-1}}$ and, thus,

$$
\mu\left(E_{\sigma(\gamma) \alpha \gamma^{-1}}\right)=\mu\left(E_{\alpha}\right), \quad \gamma \in \Gamma
$$

Since $\theta \in \operatorname{Int} R_{\Gamma}$, there is $\gamma_{0} \in \Gamma$ with $\mu\left(E_{r_{0}}\right)>0$. Consequently, the set $\left\{\sigma(\gamma) \gamma_{0} \gamma^{-1}: \gamma \in \Gamma\right\}$ is finite. Let

$$
\Gamma_{0}=\left\{\gamma \in \Gamma: \sigma(\gamma)=\gamma_{0} \gamma \gamma_{0}^{-1}\right\}=\left\{\gamma \in \Gamma: \sigma(\gamma) \gamma_{0} \gamma^{-1}=\gamma_{0}\right\}
$$

Then $\Gamma_{0}$ is a finite index subgroup in $\Gamma$. Therefore $\Gamma_{0}$ and $\sigma\left(\Gamma_{0}\right)$ are irreducible lattices in $\Gamma$. According to the Mostow-Margulis rigility theorem [Zim 4, 5.1.1], $\sigma$ is uniquely extended up to an automorphism of $G$, i. e. $\sigma(g)=$ $\gamma_{0} g \gamma_{0}^{-1}, g \in G$. In particular, $\sigma(\gamma)=\gamma_{0} \gamma \gamma_{0}^{-1}, \gamma \in \Gamma$. Consequently, $\gamma E_{\gamma_{0}}=$ $E_{\gamma_{0}}$ for all $\gamma \in \Gamma$, i. e. $\mu\left(E_{\gamma_{0}}\right)=\mu(X)$ and $\theta(x)=\gamma_{0} x$.

Now turn again to the equivalence relation $R_{\Gamma}$ generated by left translations of the group $\Gamma$ on the homogeneous space $X=K / L$. Let

$$
\begin{gathered}
\operatorname{Aut}(K, \Gamma)=\{\sigma \in \operatorname{Aut} K: \sigma(\Gamma)=\Gamma\}, \\
Q=\left\{(\sigma, t): \sigma \in \operatorname{Aut}(K, \Gamma), t \sigma(L) t^{-1}=L\right\} .
\end{gathered}
$$

For $(\sigma, t) \in Q$ the automorphism $r_{(\sigma, t)}$ of the space $(X, \nu)$ :

$$
r_{(\sigma, t)}(k L)=\sigma(k) t^{-1} L
$$

is well defined. Since $\sigma(\Gamma)=\Gamma, r_{(\sigma, t)} \in$ Aut $R_{\Gamma}$.

The next theorem gives the explicit description of the automorphisms of $R_{\Gamma}$, which is similar to [GG 2, Theorem 4.4]. We use the argument of D. Witte [Wit, 9.4] in its proof.

Theorem 3.3. Let $K$ be a connected compact simple Lie group with trivial center, $L$ its closed subgroup, and $\Gamma$ the lattice in a connected simple Lie group $G$ with trivial center, and $\mathbb{R}-\operatorname{rank}(G) \geq 2$. Suppose that $\Gamma$ is densely embedded into 
$K$, and consider the equivalence relation $R_{\Gamma}$ generated by left translations of $\Gamma$ on the homogeneous space $X=K / L$. Let $\theta \in$ Aut $R_{\Gamma}$. Then there exist $t \in K, \sigma \in$ Aut $(K, \Gamma)$ and $w \in \operatorname{Int} R_{\Gamma}$ such that $(\sigma, t) \in Q$ and $\theta=w_{(\sigma, t)}$.

Proof. According to Lemma 2.1, $\Gamma$ acts on $(X, \nu)$ freely. Consider the $G$-action induced by that of $\Gamma$. Let $\Delta: \Gamma \rightarrow K \times G$ be the diagonal embedding. Then the space of the induced action $(X \times G) / \Gamma$ can be identified with the double coset space $\Delta(\Gamma) \backslash(K \times G) / L$ (we also denote the subgroup $L \times\{e\} \subset$ $K \times G$ by $L)$. The identification is given by the map

$$
\begin{gathered}
\mathrm{id}:(X \times G) / \Gamma \rightarrow \Delta(\Gamma) \backslash(K \times G) / L, \\
\quad \operatorname{id}([x, h])=\Delta(\Gamma)(k, h) L, x=k L .
\end{gathered}
$$

Now the induced action coincides with that of $G$ by right translations on $\Delta(\Gamma)$ $\backslash(K \times G) / L($ see $(3.4))$, i. e. $g y=\Delta(\Gamma)\left(k, h y^{-1}\right) L$, where $y=\Delta(\Gamma)(k, h) L$. Let $\theta \in \mathrm{Aut}_{\Gamma}, \alpha: X \times \Gamma \rightarrow X$ be the cocycle corresponding to $\theta$ and $\widetilde{\theta}_{1} \in$ Aut $R_{G}$ the automorphism constructed while proving Proposition 3.1 (see (3.5) and (3.4)):

$$
\begin{gathered}
\tilde{\theta}_{1}([x, h])=\psi([x, h]) \tilde{\theta}([x, h])=\left[\theta\left(f(h)^{-1} x\right), s(h) \psi([x, h])^{-1}\right], \\
{[x, h] \in Y .}
\end{gathered}
$$

Then,

$$
\widetilde{\theta}_{1}(g y)=\widetilde{\sigma}_{1}(g) \tilde{\theta}_{1}(y)
$$

for all $g \in G$ and a. a. $y \in Y$. Since $(Y, \rho)$ is an ergodic $G$-space, $\widetilde{\theta}_{1}$ preserves the measure. Similarly to the proof of Corollary 9.4 [Wit], consider the following $G$-equivalent projections:

$$
\begin{gathered}
p: \Delta(\Gamma) \backslash(K \times G) / L \rightarrow \Gamma \backslash G, \\
p(\Delta(\Gamma)(k, h) L)=\Gamma h \text { and } \\
\pi_{L}: \Delta(\Gamma) \backslash(K \times G) \rightarrow \Delta(\Gamma) \backslash(K \times G) / L, \\
\pi_{L}:(\Delta(\Gamma)(k, h))=\Delta(\Gamma)(k, h) L .
\end{gathered}
$$

Taking into consideration identification (3.11), define the map $F: \Delta(\Gamma) \backslash$ $(K \times G) \rightarrow \Gamma \backslash G, F=p \circ \widetilde{\theta}_{1} \circ \pi_{L}$. In view of (3.13) $F$ is an affine map for $\{e\} \times G[$ Wit, Def. 2.2], i. e.

$$
F(\Delta(\Gamma)(k, h) g)=F(\Delta(\Gamma)(k, h)) \widetilde{\sigma}_{1}(g)
$$

at each $g \in G$ for a. a. $(k, h) \in K \times G$. Moreover, $F$ preserves the measure. 
Since $\Delta(\Gamma)$ is a lattice in $K \times G$ and $\Delta(\Gamma)$ is densely projected in $K$, in view of the reasoning given in the proof of Corollary 9.1 [Wit], $F$ is an affine map for the entire group $K \times G$, i.e.

$$
F(\Delta(\Gamma)(k t, h g))=F(\Delta(\Gamma)(k, h)) \delta(t, g),
$$

where $\delta$ is an homomorphism from $K \times G$ to $G$ with $\delta(e, g)=\widetilde{\sigma}_{1}(g), g \in G$. Hence, $\delta(t, g)=\widetilde{\sigma}_{1}(g)$ and

$$
F(\Delta(\Gamma)(k t, h g))=F\left(\Delta(\Gamma)(k, h) \widetilde{\sigma}_{1}(g)\right.
$$

at all $(t, g) \in K \times G$ for a. a. $(k, h) \in K \times G$. Consider the map $B: K \times G$ $\rightarrow \Gamma \backslash G, F(\Delta(\Gamma)(k, h)) \widetilde{\sigma}_{1}(h)^{-1}$. Using (3.14) we obtain

$$
\begin{gathered}
B(k t, h g)=F(\Delta(\Gamma)(k t, h g)) \widetilde{\sigma}_{1}(h g)^{-1} \\
=F(\Delta(\Gamma)(k, h)) \widetilde{\sigma}_{1}(g) \widetilde{\sigma}_{1}(h)^{-1}=B(k, h)
\end{gathered}
$$

for all $(t, g) \in K \times G$ and a. a. $(k, h) \in K \times G$. Consequently, there exists an element of $h_{0} \in G$ such that $B(k, h)=\Gamma h_{0}$ for a. a. $(k, h) \in K \times G$. Thus, $F(\Delta(\Gamma)(k, h))=\Gamma h_{0} \widetilde{\sigma}_{1}(h)$ for a. a. $(k, h) \in K \times G$. Furthermore, using (3.11),(3.12) and definitions of the projections $p$ and $\pi_{L}$ we obtain

$$
\begin{aligned}
F(\Delta(\Gamma)(k, h)) & =\left(p \cdot \widetilde{\theta}_{1}\right)(\Delta(\Gamma)(k, h) L) \\
& =p\left(\left[\theta\left(f(h)^{-1} x\right), s(h) \psi([x, h])^{-1}\right]\right) \\
& =\Gamma s(h) \phi([x, h])^{-1},
\end{aligned}
$$

where $x=k L \in X$. Consequently,

$$
\Gamma s(h) \psi([x, h])^{-1}=\Gamma h_{0} \widetilde{\sigma}_{1}(h)
$$

for a. a. $h \in G$ and $x \in X$. Hence, we obtain for a. a. $h=s(h) \in S$

$$
h_{0} \widetilde{\sigma}_{1}(s(h)) \psi\left([x, s(h)] s(h)^{-1} \in \Gamma\right. \text {. }
$$

Set up $\phi_{1}([x, h])=h_{0} \widetilde{\sigma}_{1}(s(h)) \psi([x, s(h)]) s(h)^{-1}$. Then

$$
\phi([x, s(h)])=\widetilde{\sigma}_{1}(s(h))^{-1} h_{0}^{-1} \psi_{1}([x, s(h)]) s(h)
$$

and $\phi_{1}([x, h]) \in \Gamma$ for a. a. $x \in X$ and $h \in G$. Now substitute (3.15) into (3.10) for the cocycle $\alpha$ (see the Proof of Proposition 3.1): 


$$
\begin{aligned}
\alpha(x, \gamma) & =s(h) \phi([\gamma x, s(h)])^{-1} \widetilde{\sigma}_{1}\left(s(h)^{-1} \gamma s(h)\right) \psi([x, s(h)]) s(h)^{-1} \\
& =\phi_{1}([\gamma x, s(h)])^{-1} h_{0} \widetilde{\sigma}_{1}(\gamma) h_{0}^{-1} \phi_{1}([x, s(h)])
\end{aligned}
$$

for all $\gamma \in$ and a. a. $x \in X$ and $h \in G$.

Fix the element $s(h) \in S$ such that (3.15) is satisfied for a. a. $x \in X$ and set up

$$
\eta(x)=\phi_{1}\left([x, s(h)], \sigma_{0}(\gamma)\right)=h_{0} \widetilde{\sigma}_{1}(\gamma) h_{0}^{-1}
$$

Then for all $\gamma \in \Gamma$ and a. a. $x \in X$ we obtain

$$
\alpha(x, \gamma)=\eta(\gamma x) \sigma_{0}(\gamma) \eta(x), \eta(x) \in \Gamma \text { and } \sigma_{0} \in \operatorname{Aut} \Gamma
$$

since $\sigma_{0}(\gamma)=\eta(\gamma x) \alpha(x, \gamma) \eta(x)^{-1} \in \Gamma$.

Now let $\theta_{1}(x)=\eta(x) \theta(x)$. Then $\theta_{1} \in$ Aut $R_{\Gamma}$ and

$$
\theta_{1}(\gamma x)=\sigma_{0}(\gamma) \theta_{1}(x)
$$

Let us show that $\sigma_{0} \in \operatorname{Aut} \Gamma$ can be extended up to an automorphism $\sigma \in$ Aut $(K, \Gamma)$. Consider the representation $U$ of $K$ in the space $L^{2}(X, \nu)$ :

$$
\left(U_{k} \xi\right)(x)=\xi\left(k^{-1} x\right), \quad k \in K, \quad x \in X=K / L .
$$

Define also the unitary operator $V$ :

$$
(V \xi)(x)=\xi\left(\theta_{1}(x)\right), \quad x \in X
$$

Then from (3.16) we obtain that

$$
U \gamma V=V U_{\sigma_{0}(r)} \text { or } U_{\sigma_{0}(\gamma)}=V^{*} U_{\gamma} V, \gamma \in \Gamma
$$

Set up $U_{\sigma(k)}=V^{*} U_{k} V, k \in K$. Since $U$ is a strongly continuous representation and $\Gamma$ is a dence subgroup in $K, U_{\sigma(k)} \in\left\{U_{t}: t \in K\right\}$. Thus, $\sigma$ is an automorphism of $K$ and $\sigma(\gamma)=\sigma_{0}(\gamma)$ for $\gamma \in \Gamma$, i.e. $\sigma \in$ Aut $(K, \Gamma)$. Furthermore, consider the Borel map

$$
\beta: K \rightarrow X, \beta(k)=\sigma(k)^{-1} \theta_{1}(k L)
$$

In view of (3.16) 


$$
\beta(\gamma k)=\sigma\left(\gamma_{k}\right)^{-1} \theta_{1}(\gamma k L)=\beta(k)
$$

for all $\gamma \in \Gamma$ and a. a. $k \in K$. Consequently, there exists an element $t \in K$ such that $\beta(k)=t^{-1} L$, i.e. $\theta_{1}(k L)=\sigma(k) t^{-1} L$ for a. a. $k \in K$. We show that $t \sigma(L) t^{-1}=L$. Let $l \in L$. Then there is $k \in K$ with $\theta_{1}(k L)=\sigma(k) t^{-1} L$ and $\theta_{1}((k l) L)=\sigma(k l) t^{-1} L$. Therefore $\sigma(k) t^{-1} L=\sigma(k l) t^{-1} L$, i.e. $\quad t \sigma(l) t^{-1} \in L$. Consequently, $t \sigma(L) t^{-1} \subset L$. In view of the compactness of $L$ we obtain $t \sigma(L)$ $t^{-1}=L$. Thus, $\theta_{1}=r_{(\sigma, t)}$, where $(\sigma, t) \in Q$. Now for $w=\theta \theta_{1}^{-1}$ we obtain $w$ $\in \operatorname{Int} R_{\Gamma}$ and $\theta=\operatorname{wr}_{(\sigma t)}$.

Corollary 3.4. Let all the conditions of Theorem 3.3 are satisfied. Then the group Out $R_{\Gamma}$ is compact. Moreover, if $L$ is a finite index in $N_{K}(L)$, Out $R_{\Gamma}$ is finite.

Proof. Set up $H_{1}=N_{K}(L) / L, H_{2}=N_{K}(\Gamma) / \Gamma$. Using the explicit form of the automorphism from Aut $R_{\Gamma}$ obtained in Theorem 3.3 and Lemma 2.6, it can be readily verified that $H_{1}$ and $H_{2}$ are embedded into Out $R_{\Gamma}$ as normal subgroups. Besides, in view of Lemma $3.2 H_{1} \cap H_{2}=\{e\}$ in Out $R_{\Gamma}$. Let us show that the group $\left(\left(\right.\right.$ Out $\left.\left.R_{\Gamma}\right) / H_{1}\right) / H_{2}$ is finite. If $\left(\sigma_{1}, t_{1}\right),\left(\sigma_{2}, t_{2}\right) \in Q$, the automorphisms $\gamma_{\left(\sigma_{1}, t_{1}\right)}$ and $r_{\left(\sigma_{2}, t_{2}\right)}$ differ by the right translation $r_{t_{0}}$, where $t_{0}=t_{1} t_{2}^{-1} \in N_{k}(L)$. Further, Out $K$ is a finite, therefore $N_{k}(\Gamma)$ is a finite index subgroup in Aut ( $K$, $\Gamma)$ (remind that $Z(K)=\{e\})$. Now the finiteness of $\left(\left(\right.\right.$ Out $\left.\left.R_{\Gamma}\right) / H_{1}\right) / H_{2}$ follows from the fact that each element of Out $R_{\Gamma}$ is the image of $r_{(\sigma, t)}$ where $(\sigma, t) \in Q$ (Theorem 3.3). To complete the proof, it suffices to show that the $H_{2}$ is finite. Since $Z(K)=Z(\Gamma)=\{e\}$, we have that $H_{2}$ is embedded into Out $\Gamma$. However, it follows from the finiteness of Out $G$ ([Bou, Ch. III, Section 9, Prop. 30 (ii)] and $[$ Mos, p. 254] $), N_{G}(\Gamma) / \Gamma([\mathrm{Rag}, 5.17])$ and the Mostow-Margulis rigidity theorem $([\operatorname{Zim} 4,5.1 .1])$ that Out $\Gamma$ is finite.

Corollary 3.5. Assume that Aut $K=\operatorname{Int} K, N_{k}(L)=L$ and $N_{k}(\Gamma)=\Gamma$. Then Aut $R_{\Gamma}=\operatorname{Int} R_{\Gamma}$, i. e. Out $R_{\Gamma}$ is trivial.

Proof. Let $\theta \in \operatorname{Aut}_{\Gamma}$. According to Theorem 3.3, $\theta=w r_{(\sigma, t)}$, where $w \in$ $\operatorname{Int} R_{\Gamma}$ and $r_{(\sigma, t)}=\sigma(k) t^{-1} L$ with $\sigma \in \operatorname{Aut}(K, \Gamma)$ and $t \sigma(L) t^{-1}=L$. Since Aut $K$ $=$ Int $K$, we have $\sigma(k)=k_{0} k k_{0}^{-1}, k_{0} \in K$. Hence, $k_{0} \in N_{k}(\Gamma)=\Gamma, t k_{0} \in N K(L)=$ $L$, and $r_{(\sigma, t)}(k L)=k_{0} k k_{0}^{-1} t^{-1} L=k_{0} k\left(t k_{0}\right)^{-1} L=k_{0} k L$, i. e. $r_{(\sigma, t)} \in \operatorname{Int} R_{\Gamma}$. Consequently, $\theta=w r_{(\sigma, t)} \in \operatorname{Int} R_{\Gamma}$. 


\section{§4. An Example of Ergodic Equivalence Relation without Outer Automorphisms}

Let us proceed to the construction of an example of an equivalence relation without outer automorphisms.

Consider the quadratic form

$$
f(x)=x_{1}^{2}+x_{2}^{2}+\cdots+x_{n-2}^{2}-\sqrt{2} x_{n-1}^{2}-\sqrt{2} x_{n}^{2}
$$

where $n=2 l+1 \geq 5$. Let $G$ be a connected component of the identity in $S O$ $(f, \mathbf{R})$ and $\Gamma_{0}=S O(f, \mathbb{Z}[\sqrt{2}]) \cap G$. Then $G$ is a connected simple Lie group with trivial center, $\mathbb{R}-\operatorname{rank}(G)=2$, and $\Gamma_{0}$ is a lattice in $G$ (see $[Z$ im $4,5.2 .12$ and 6.1.5] and [Sul]). Denote the nontrivial automorphism of the field $\mathbf{Q}(\sqrt{2})$ by $\tau: \tau(a+b \sqrt{2})=a-b \sqrt{2}$.

Consider now the quadratic form

$$
f^{\tau}(x)=x_{1}^{2}+x_{2}^{2}+\cdots+x_{n-2}^{2}+\sqrt{2} x_{n-1}^{2}+\sqrt{2} x_{n}^{2}
$$

and let $K=S O\left(f^{\tau}, \mathbf{R}\right)$. Then $K$ is a connected compact group which is isomorphic to $S O_{n}(\mathbf{R})$ and the group $S O(f, \mathbb{Z}[\sqrt{2}])$ is embedded into $K$ via $\tau$ as a dense subgroup [Sul]. Since $\Gamma_{0}$ is a finite index subgroup in $S O(f, \mathbb{Z}[\sqrt{2}])$ it is also densely embedded into $K$. We will identity $\Gamma_{0}$ with its image in $K$.

\section{Lemma 4.1 .}

$$
\begin{array}{ll}
\text { (1) } & N_{k}\left(\Gamma_{0}\right) \subset S O\left(f^{\tau}, \mathbf{Q}(\sqrt{2})\right) ; \\
\text { (2) } & N_{G}\left(\Gamma_{0}\right) \subset S O(f, \mathbb{Q}(\sqrt{2})) .
\end{array}
$$

Proof. We prove (1). Set up $H=S O\left(f^{\tau}, \mathbf{C}\right)$. Then $H$ is a simple algebraic group defined over the field $\mathbf{Q}(\sqrt{2})$. Let $k \in N_{k}\left(\Gamma_{0}\right)$. Since $\Gamma_{0}$ is dense in $K$, and $K$ is Zariski dense in $H, \Gamma_{0}$ is also Zariski dense in $H$. Therefore the inner automorphism $h \rightarrow k h k^{-1}$ of $H$ is defined over $\mathbb{Q}(\sqrt{2})$ [Zim 4, 3.1.10]. Consequently, $\operatorname{Ad} k \in(\operatorname{Ad}(H))_{Q(\sqrt{2})}$, where $\operatorname{Ad}(H)$ is the adjoint group. Since the center of $H$ is trivial, $\mathrm{Ad}: H \rightarrow \operatorname{Ad}(H)$ is an isomorphism defined over $\mathbf{Q}(\sqrt{2})$. Consequently,

$$
k \in H_{Q(\sqrt{2})}=S O\left(f^{\tau}, \mathbf{Q}(\sqrt{2})\right) .
$$

Similarly proposition (2) is proved.

Set up $\Gamma_{1}=N_{G}\left(\Gamma_{0}\right)$. Then $\Gamma_{1}$ is a discrete subgroup and, hence, a lattice in $G$ [Rag, 5.17]. Besides, $\Gamma_{1} \subset S O(f, \mathbf{Q}(\sqrt{2}))$. In view of Lemma 4.1 we can 
identify $\Gamma_{1}$ with $N_{K}\left(\Gamma_{0}\right)$ via $\tau$. For $\Gamma_{1}$ there are also embeddings

$$
\begin{aligned}
& N_{k}\left(\Gamma_{1}\right) \subset S O\left(f^{\tau}, \mathbf{Q}(\sqrt{2})\right) \text { and } \\
& N_{G}\left(\Gamma_{1}\right) \subset S O(f, \mathbf{Q}(\sqrt{2})) .
\end{aligned}
$$

Continuing this construction, we obtain the sequence of lattices $\left\{\Gamma_{m}\right\}$ in $G$ with $\Gamma_{m+1}=N_{G}\left(\Gamma_{m}\right)$. Moreover, all the groups $\left\{\Gamma_{m}\right\}$ are embedded into $K$ via $\tau$ as dense subgroups, and we have $\Gamma_{m+1}=N_{K}\left(\Gamma_{m}\right)$. According to [Rag, 9.8], there is $m_{0}$ with $\Gamma_{m}=\Gamma_{m_{0}}$ for all $m \geq m_{0}$. Put $\Gamma=\Gamma_{m_{0}}$. Then $N_{K}(\Gamma)=\Gamma$. Further more, identifying $K$ with $S O_{n}(\mathbb{R})$, we denote by $L$ the subgroup in $S O_{n}(\mathbb{R})$ consisting of matrices of the form

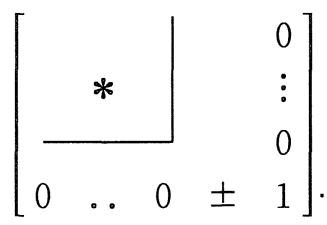

Consider the $\Gamma$-action on the homogeneous space $X=K / L$.

Theorem 4.2. All the automorphisms of the equivalence relation $R_{\Gamma}$, are inner.

Proof. According to [Die, ch. IV], Aut $K=\operatorname{Int} K$. Let us show that $N_{k}(L)$ $=L$. Indeed, consider the projection $p=$

$$
=\left[\begin{array}{cccc} 
& & 0 \\
& 0 & & \vdots \\
& & & 0 \\
\hline 0 & \ldots & 0 & 1
\end{array}\right] .
$$

Then

$$
L=\left\{k \in K: k p k^{-1}=p\right\}
$$

If $k \in N_{K}(L)$, then for $l \in L$ we have $k l k^{-1} p=p k l k^{-1}$, i. e. $l\left(k^{-1} p k\right)=\left(k^{-1} p k\right) l$. Hence, $k^{-1} p k=p$, i. e. $k \in L$. Thus, all the conditions of Statement 3.5 are satisfied.

Corollary 4.3. Let $\mathrm{I}_{\infty}$ be the transitive equivalence relation on $(\mathbb{Z}, \delta)$ where $\delta(\{n\})=1$. Then all the automorphisms of the $\mathrm{II}_{\infty}$-equivalence relation $\widetilde{R}_{\Gamma}=R_{\Gamma}$ 


\section{$\times \mathrm{I}_{\infty}$ are inner.}

Proof. According to [GG 2, Corollary B.3] all the automorphisms of Aut $\widetilde{R}_{\Gamma}$ preserve the measure. Therefore, the embedding of Aut $R_{\Gamma}$ into Aut $\widetilde{R}_{\Gamma}, \theta \rightarrow$ $\theta \times$ id generate an isomorphism of Out $R_{\Gamma}$ and $\operatorname{Aut} \widetilde{R}_{\Gamma}$.

\section{References}

[Bou] Bourbaki, N. Groupes et algébres de Lie, Hermann, Paris, 1971.

[Cho 1] Choda, M., Crossed product and property T, Math. Jap., 26 (1981), 557-567.

[Cho 2] - A condition to construct a full $I_{1}$-factor with an application to approximate normalcy, Math. Jap., 28 (1983), 383-398.

[Con 1] Connes, A., Almost periodic states and factors of type III 1 J. Func. Anal., 16 (1974), 415-445.

[Con 2] - A factor of type $\mathrm{I}_{1}$ with countable fundamental group, J. Operator Theory, 4 (1980), 151-153.

[CFW] Connes, A., Feldman, J. and Weiss, B., An amenable equivalence relation is generated by a single transformation, Ergod. Theory Dyn. Syst., 1 (1981), 431-450.

[Dan] Danilenko, A,I., The topological structurre of Polish groups and groupoids of measure space transformations, Publ. RIMS, Kyoto Univ., 31 (1995), 913-940.

[Die] Dieudonné, J., La géométrie des groupes classiques, (Springer-Verlag, Berlin-HeildeibergNew York, 1971).

[EW] Enomoto, M., Watatani, Y., Some remarks of $\mathbb{I}_{1}$-factors whose inner automorphism groups are open, Math. Jap., 26 (1981), 254-247.

[FM 1] Feldman, J. and Moore, C.C., Ergodic equivalence relations, cohomology, and von Neumann algebras I, Trans. Amer. Math. Soc., 234 (1977), 289-324.

[FM 2] - Erogodic equivalence relations, cohomology, and von Neumann algebras II , Trans. Amer. Math. Soc., 234 (1977), 325-359.

[GGN] Gefter, S.L., Golodets, V.Ya. and Nessonov, N.I., Actions of T-groups on Von Neumann algebras and factors with coutable fundamental groups, Functional Anal. Appl., 119 (1985), 64-65.

[GG 1] Gefter, S.L., Golodets, V.Ya., Ergodic action with the identity fundamental group, Dokl. UkrSSR Acad. Sci., 10(1986), 8-10.

[GG 2] - Fundamental groups for ergodic actions and actions with unit foundamental groups, Publ. RIMS, Kyoto Univ. 24(1988), 821-847.

[Gef] Gefter, S.L., Ergodic equivalence relation without outer automorfisms, Dokl. Acad. Sci. of Ukraine, 11 (1993), 25-27.

[Gol] Golodets, V.Ya., Actions of T-groups on Lebesgue spaces and properties of full factors of type $\Pi_{1}$, Publ. RIMS, Kyoto Univ., 24 (1986), 821-847.

[Haa] Haagerup, U., The standard form of von Neumann algebras, Math. Scand., 37 (1975), 271-283.

[HO] Hamachi, T., Osikawa, M., Fundamental homomorphism of normalizer group of ergodic transformation. Lect. Notes Math., 729 (1979), 43-57.

[JS] Jones, V.F.R., Schmidt, K., Asymptotically invariant sequences and approximate finiteness, Amer. J. Math., 109 (1987), 91-114. 
[Kaz] Kazhdan, D.A., Connection of the dual space of a group with the suructure of its closed subgroups, Functional Anal. .Appl., 1 (1967), 63-65.

[Mos] Mostow, G.D., Fundamental groups of homogeneous spaces, Ann. of Math., 66 (1957). 249-255.

[Rag] Raghunthan, M.S., Discrete subgroups of Lie groups, Springer-Verlag, Berlin-HeidelbergNew York, 1972.

[Sak] Sakai, S., On automorphism groups of $\Pi_{1}$-factors, Tohoku Math. J., 26 (1974), 423-430.

[Sul] Sullivan, D., For $n>3$ there is only one finitely additive rotationally invariant measure on the $n$-sphere defined on all Lebesgue measurable subsets, Bull. Amer. Math. Soc., 4 (1981), 121-123.

[Wan] Wang, S.P., On density properties of $S$-subgroups of locally compact groups, Ann. Math., 94(1971), 325-329.

[Wit] Witte, D., Rigidity of some translataions on homogeneous spaces, Invent. Math., 81 (1985). $1-27$.

[Zim 1] Zimmer, R.J., Induced and amenable ergodic actions of Lie groups, Ann. Ecole Norm. Sup., 11 (1978), 407-428.

[Zim 2] Strong rigidity for ergodic actions of semisimple Lie groups, Ann. of Math., $112(1980), 511-529$.

[Zim 3$]$ - Orbit equivalence and rigidity of ergodic actions of Lie groups, Ergod. Theory Dyn. Syst., 1 (1981), 237-253.

[Zim 4 ] - Ergodic theory and semisimple groups, Boston, Birkhäuser, 1984. 